\title{
Generation of two-lobe light fields with a rotating intensity distribution under propagation for single emitter spectroscopy
}

\author{
V.G. Volostnikov ${ }^{1,2}$, E.N. Vorontsov ${ }^{1}$, S.P. Kotova ${ }^{1,2, *}$, N.N. Losevsky ${ }^{1}$, D.V. Prokopova ${ }^{1,2}$, \\ E.V. Razueva ${ }^{1}$, and S.A. Samagin ${ }^{1}$ \\ ${ }^{1}$ Samara Branch of the Lebedev Physical Institute RAS, 443011 Samara, Russia \\ ${ }^{2}$ Samara National Research University, 443086 Samara, Russia
}

\begin{abstract}
The paper outlines the results of studies on the generation of twolobe light fields with the intensity distribution rotating during the field propagation. Such fields are needed to determine the depth of bedding of single emitters in spectral studies of substance properties. On the base of the spiral beam optics, the phase distributions were obtained for the synthesis of two-lobe fields with different speeds of rotation of the intensity distribution. The light fields have been formed by using a liquid-crystal spatial phase modulator HOLOEYE HEO-1080P. The influence of the illuminating beam parameters and the aberrations of the system on the quality of the formed light field was also studied.
\end{abstract}

The problem of accurately determining the position of single fluorescent molecules, quantum dots and other nanostructures arises in the spectral study of physical and chemical properties of substances [1,2]. Meanwhile the point light source coordinates in space are likely to change with time. The task of the exact estimation of the longitudinal coordinate of the emitting nano-size object in microscopy is still unsolved. As a part of this task focuses on the use of light fields on the distribution of the intensity of which can determine the depth of the object. And the two-lobe light fields with their intensity distribution looking like two maxima, are considered to be particularly attractive ones [3].

The idea of the suggested approach for estimating the depth of bedding is as follows. The radiation from the point light emitters passes through the optical system of a microscope and a special phase filter. Essentially this filter is a diffraction optical element that transforms a plane wave field into a field with two maxima in the intensity distribution profile (the two-lobe field) rotating in the course of propagation. Therefore, within the observation plane the image of the point object looks like two high intensity peaks. The object longitudinal displacement can be evaluated by the change of the intensity maxima position within the observation plane. And the higher the rotation velocity of the intensity of the field generated by the diffraction element is, the more accurate the estimation of the longitudinal coordinate of the emitting object can be. By the rotation velocity we mean the

* Corresponding author: kotova@fian.smr.ru 
value that determines the rotation angle of the intensity distribution of the light field under its propagation in the longitudinal direction.

The paper is dedicated to the study of spiral-optics-based generation of the phase optical elements that form two-lobe light fields with the intensity distribution rotating at their propagation. Discovered in nineties of the XX-th century, the spiral beams were wellstudied [4]. Spiral beams are paraxial light fields with a transverse intensity distribution which rotates around the beam axis maintaining its shape (but not the scale) whilst propagating. It was shown that the spiral beams could be constructed so that to obtain the field with predetermined intensity distribution and rotation parameters. The expression defining the rotation angle $\theta$ of the spiral beam intensity in the plane located at $\mathrm{z}$-distance from the initial plane is as follows:

$$
\theta(z)=\theta_{0} \arctan \left(\frac{2 z}{k \rho^{2}}\right),
$$

where $\mathrm{k}$ is a wave number $\rho$ - parameter describing the beam lateral dimension, $\theta_{0}-$ parameter describing the beam rotation velocity during its propagation. A full angle of the beam rotation from the waist to a remote diffraction zone is:

$$
\theta=\theta_{0} \frac{\pi}{2}
$$

Two approaches were used to obtain phase distributions for the generation of two-lobe fields with different rotation velocity of the intensity distribution. In the first one the initial spiral beam is a sum of several Laguerre-Gauss modes [5], in the second one it was obtained by functional representation [6]. Phase filters were developed for the generation of fields with the rotation parameters $\theta_{0}=-1, \theta_{0}=-2$ and $\theta_{0}=-4$. By numerical modeling, parameters of the field illuminating the phase mask were determined for the most effective generation of the light fields under research and were used in experiments conducted.

The experiments on generation of two-lobe light fields were fulfilled with the phase spatial liquid crystal modulator of HOLOEYE HEO-1080 type. Obtained dependencies for the rotation angle vs distance well agreed with the theoretical ones. Note that the energy effectiveness went down with the increase of $\theta_{0}$ value. For the experimentally obtained light fields with the highest rotation velocity it was not exceeding $30 \%$, while for the case of $\theta_{0}=-2$ it achieved its maximum value of $56 \%$.

A number of experiments on evaluation of the effect of coma, astigmatism and spherical aberrations on two-lobe light fields were realized. It was discovered that for the values of $0,198 \lambda, 0,177 \lambda$ and $0,153 \lambda$ for spherical aberrations, coma and astigmatism respectively, it was still feasible to get the information on the angular position of the two maxima of the field portions.

The support from Russian Foundation for Basic Research is acknowledged (16-2911809).

\section{References}

1. A.V. Naumov, Phys. Usp. 56, 605 (2013)

2. A.V. Naumov, I.Yu. Eremchev, A.A. Gorshelev, Eur. Phys. J. D 68, 348 (2014)

3. S.R.P. Pavani, R. Piestun, Opt. Express 16, 3484 (2008)

4. E.G. Abramochkin, V.G. Volostnikov, Modern Optics of Gaussian beams (Moscow, Fizmatlit, 2010)

5. V.G. Volostnikov, E.N. Vorontsov, S.P. Kotova, N.N. Losevsky, D.V. Prokopova, Bull. Ras: Physics 80, 766 (2016)

6. E. Razueva, E. Abramochkin, EPJ Web of Conferences 103, 10011 (2015) 